\title{
The Syntactic Role Of Colour in Film
}

\author{
By Ornam Rotem \\ Spring 2003 Issue of KINEMA
}

\section{THE WORLD AS ONE WHOLE: THE SYNTACTIC ROLE OF COLOUR IN FILM}

JEAN RENOIR greatly admired Marcel Pagnol, but unlike him, he did not believe "that everything was in dialogue". As a film-maker Renoir sought to combine the disparate elements that make up a film, each finding its own place in the grand scheme. He said that in a given situation, say a love-scene when two adoring actors approach one another, "dialogue is a part of the theme and reveals character" but the true weight of emotions are carried by other, non-verbal factors, mostly those revealed in close ups. Moreover, the real theme in this example (of a love-scene), is the person. Dialogue, texture, picture, situation, setting, temperature and lighting all combine together to depict the person, since, as he puts it, "the world is one whole".(1)

There is something uncanny about Renoir when he philosophizes. Like his films, the rambling and colloquial style may cause one to overlook his formidable depth. This last interjection about the world and its wholeness comes as if from nowhere. He seems to be talking about the nature of film and this is certainly the context, but then, having enumerated its constituent elements, he suddenly concludes with what is clearly a non sequitur: "The world is one whole". We should not hold this apparent fallacy against him since it is Renoir the poet talking here, and poets are licensed to be loose with logics. What I take him to be suggesting is that when the real theme is the person, the different elements that make a film do not merely combine to become a film, they combine to become a world, a whole world and the world as a whole. Admittedly, in this scheme of things different elements serve different (yet overlapping) purposes, but I shall argue that one element is crucial to creating a sense of whole. Renoir has enviously referred to it as "temperature and lighting", but I will resort to a more prosaic term: colour. In what follows I will explore the role colour has in the formation of a film world and demonstrate this by a detailed look into the chromatic structure of Hitchcock's Vertigo.

To begin with, it is imperative to make few clarifications. In everyday language, we use colour to signify (at least) two separate, but inter-related concepts. On the one hand, colour has a specific sense, as in the question "what is the colour of his car?" The list of possible hues is the list of possible answers: red, blue, green, yellow, pink, etc. Strictly speaking, we should be asking what is the hue of his car, but this is not common practice in everyday language. Similarly, we use colour in contradistinction to "black and white" supposedly implying that the latter is void of colour. The other, and more precise, use of the term "colour", refers to that attribute of things that causes the light they reflect to produce a certain kind of visual sensation. When someone informs us that he dreams in colour he is reporting to us about a certain quality of his visual experience and we should not hold it against him if he cannot recall the specific hue of the car he was dreaming about. By the same token, if one thinks of Paul Klee's admission that colour and he are one, it is evident that he is referring to a much broader mental category than the hues on his palate.

Colour possesses me. I don't have to pursue it. It will possess me always, I know it. That is the meaning of this happy hour: Colour and I are one. I am a painter. ${ }^{(2)}$

In my use of the term colour I will be referring to that decisive attribute of visual experience, the stuff out of which visual phenomena are made and which comprises one of the most important keys in our ability to organize visual space. In this capacity colour should be seen as the combination of subjective and objective determinants quantified in three dimensions, namely, as varying hues, as degrees of intensity or saturation and as levels of brightness.

For our purposes let us take colour to mean the whole gamut of experiences that determine visual experience, be it represented monochromatically or even as mere variances between dark and light. In cinematic terms, colour is the displacement of light on the screen and this occurs in the three dimensions mentioned above (hue, saturation and brightness). Films can express themselves in all three dimensions, at times emphasizing or eliminating others. 
The next term that needs to be clarified when exploring the role of colour in film is the concept of a world, since it is my contention that colour, in all its dimensions, plays a crucial role in being able to give a film a sense of whole and accordingly, a sense of coherence that in unison identify the exact grounding of a filmic world and determine our ability to enter this world, to be moved by it and be enlightened through it. Generally speaking, a world is construct made of inter-related and inter-dependent parts intuitively grasped.

There is a multiplicity of worlds and we talk of them in many different contexts. In order to distinguish between the many different worlds that populate our discourse, we usually index them by an adjective or clinch them in genitive formation: the material world, the sensual world, the world of pure space, the world of Form (Platonic Ideas), etc. Different worlds can overlap and interact and they need not have well defined boundaries. Works of art can be considered as worlds, as can genres or whole media. Worlds can be imaginary or imagined. Opposing this pluralism one might imagine a monism that claims that there is but one world. There is however one world that stands out from all other, one world that serves as a reference point for all other worlds and this world we qualify as the real world.

This trivial claim is difficult to uphold philosophically and has generated endless and heated discussions from the dawn of philosophy. Different epochs and different cultures have worked with very different understandings of what the real world is and thus from a comparativist point of view we are forced to conclude that perhaps the idea of a real world is not an enduring entity but rather an aspiration, since there is so little agreement about what is the nature of such real world. Be that as it may, there is little doubt that within any one scheme, the real world, however understood, holds a pivotal role in determining and appraising all other worlds. Invariably the real world has an attested unifying element. Understandably, these unifying elements differ from each other as much as the worlds themselves differ. For a scientist espousing strict materialism, the unifying element of the real world is matter and causality, for a theologian, it is God.

A filmic world is the coherent combination of its constituent elements such that it gives rise to a recognizable whole to which we can relate and respond. The presiding metaphors identifying the presence of a filmic world are terms denoting entrance to, or penetration of this world. It is as if the speaker is saying that the filmic artifice is powerful enough to generate a material presence such that he or she can wholly submit to its overriding perceptual authority. No doubt that among the arts, film is among those media (like opera) that blatantly demand the full surrender of one senses and the mind's undivided attention. Throughout its history it exhibits an ever growing tendency to draw more of the spectator's attention. Besides issues in film exhibition there are internal features of film that embrace the spectator. The most familiar, and certainly the most celebrated, is the sense of wholeness and continuity arising from narrative.

And yet, so I contend, a key unifying element in film-world is colour, the gentle balancing of light and dark and the multifarious use of different sets of hues suspended in varying intensities as they coalesce to convince the mind's eye to surrender itself. The move to identify colour as part of the deep structure of a filmic world is a conscious move away from the predominance of narrative, a move into its more subtle structures. This exploration is crucial to the process of philosophizing film since without it a crucial voice in which film speaks is enfeebled, if not entirely silenced.

Colour works two ways in film. It can serve as an internal "syntactic" feature, generating a sense of continuity, wholeness and an overall unity. It can also have a corresponding external feature - a semantic one - that designates the symbolic import of the different hues and their combinations. Much has been said about the semantics of colour, but the most thorough historical analyses inevitably conclude that colours have no universal meanings and that their semantics are determined by their social and historical import (consider, for example, John Gage's Colour and Culture). In what follows I am less interested in establishing the semantics of colour, what colours mean, but rather how colour creates a spatio-temporal framework that generates a sense of wholeness and continuity. This latter quality is evidenced in colour's syntactic qualities. The syntactic use of colour is more crucial to the formation of a film-world than its semantics. This claim does not amount to a complete rejection of an investigation of the symbolic meaning of hues, merely relegating it a less central position.

Not every film explores the medium's full potential and there are many instances of film that rely mainly, or solely, on narrative for a sense of coherence; such films are disparagingly referred to as "photographed novels" or "filmed theatre". Nevertheless, film at it best makes ample us of the means at its disposal and this 
patently obvious in the syntactic use of colour. It is worth noting that some filmmakers are acutely aware of this. As early as 1942, long before his first features and even before his first short film, Michelangelo Antonioni wrote an article on colour in film entitled "Suggestions from Hegel". He takes Hegel's idea of colour as a kind of echo and sees it in cinema as a train of reverberations, "a changing of tone being caught up and carried over else where, in play of tones created for example by the passage of clouds over the valley in John Ford's Stagecoach." (3) These ideas are further elaborated in a treatment for a film he wrote the same year in which he states that use of colour in that film will neither be exhibitionism nor décor, it will meet the needs of the narrative, "colour will determine not only the climate, but [also] the psychological movement, the drama, which involves - visually - the changing of colours". (p. 162)

Not surprisingly, when twenty-four years later he finally made a film in (spectral) colour, Il deserto rosso, he exercised such intense control over colour that grass, buildings, objects and even entire woods were painted. Sam Rohdie has quite rightly identified this as a film in which colour appears as a subject in its own right, "[Colour] is connected to the narrative but also autonomous from it, an area in which narrative loses itself in abstraction, and a new subject, the colour, the 'writing' of the film seems to displace it". (p. 155) Lastly, we need to note the discrepancy between colour and the way we talk about colour. The number of different colours we perceive is in the range of several millions and we are endowed with the ability to discern the minutest variances in colour. At the same time, in any given language the number of basic colour terms is barely eleven. Though this has been thoroughly documented, the so-called puzzle of colour-terminology remains: "why such a rich human experience of colour has issued such a universally impoverished vocabulary". (4)

Maybe the real puzzle is the fact that it puzzles us, the fact that we expect different experiences of the world to be somehow commensurable. This kind of metaphysical conceit should caution us with regard to what one may hope for in film. Just as it is hopeless looking for a commensurability between visual perception and language, so too, when looking at film-worlds, it would be wrong to aspire for a similar kind of unattainable proportion between a film's visual import, represented here by colour, and the film's verbal content, typically manifested through its narrative.

In what follows it is crucial not to regard the film's use of colour as a simplistic coding system or as a perfunctory indexing device. Colour is part of a film's internal language and while it is possible to expose its underlying structures, its basic grammar. At times one can feel a bit envious of the Bellona Islander in Polynesia who told the team of Danish anthropologists who came there to investigate colour, "We don't talk much about colour here". (5)

So, let us talk about colour. It has often been remarked that the use of colour in Vertigo is masterful; I take that to be a given. I would like to convince you that this masterful use of colour ties in with our current theme, namely, that of the construction of precise film worlds, and that these worlds are made of chromatic building blocks. When the different dimensional elements of colour (hue, saturation and brightness) converge to form a recognizable structure within a film, I shall refer to it as a chromatic schema. This schema is made apparent by the prevalence and recurrence of the colour elements that comprise the schema. Thus, for example, in Kieslowski's Trois couleurs: Bleu different shades of primary blue run through the film appearing in objects, or as reflections, or in colour casts and even through verbal reference (consider the film's title) such that taken together these varied occurrences of blue form a chromatic schema. Chromatic schemata in turn give rise to a set of coherent and narratable conditions which constitute discernable worlds. In the example of Bleu, it is evident to anyone that this chromatic structure forms a recognizable world. What exactly this world is and how to demarcate its precise boundaries calls for a different procedure. We may decide to call it by a name, but it is obvious that this will merely be a contingent label.

When we consider colour as the underlying framework of a film world, when we venture beyond its immediate cognitive effectuations so as to uncover its structural properties, we find a set of theoretical relations that function as an organizing principle mediating between the film and its spectator. For clarity's sake this may be likened to a psycho-dynamic theory of personality. A person is a person; he is not reducible to the combination of his id, ego and super-ego. Freud, in introducing this tripartite structural account, is not offering a material analysis of the mind, but rather a meta-psychology of general concepts. For example, the id is not a reification of the mind's unconscious instinctual trends, but an attempt to offer an organizing 
theoretical principle that the therapist can implement in his or her analysis of the patient and that, moreover, functions as one element of a full-fledged general theory of personality. Similarly, chromatic schemata are an organizing principle located in the space between one's apperception of the film and the film as a creation in its own right. As a set of theoretical relations colour bridges the perceptive act of watching a film and the apperceptive act of comprehending and assimilating the film in our mind. In philosophical terms, the film we are watching is a world and thus the object of apperception is not sense data, but a theoretical construct evidencing a higher degree of organization than sense data.

The theoretical construct by which we can identify a film world is the film's chromatic schemata. Colour in this instance is not a mental construct, but a reflection on the construction of the mental. Colour in the "regular", cognitive or perceptual sense, is a mental phenomenon grounded in the physics and psychobiology of how we perceive the external world. It is a feature of our visual sense and since colour itself is not only a physical property, it is obviously a complex mental construct (namely, the differential coding of a section of the electromagnetic spectrum). On the other hand, colour as an organizing principle in the apperceptive recognition and comprehension of a film world is a theoretical idea lodged in between the sensual and the intellectual. From the sensual point of view it is a mental construct, a feature of a higher degree of organization. From the intellectual point of view, colour is evidently sensual since it is lodged in the realm of perception.

A filmic colour schema displays a higher degree of colour organization than colour, by virtue of the fact that it less elemental. Schemata will inevitably reflect this higher organization and thus we are forced to conclude that in film, colour is not only a mental construct but also a reflection on the construction of the mental. It is evident, for example, that the eye perceives the film Vertigo, that strip of celluloid running through the projector, in colour. More subtle is the process by which the apperceiving mind has Vertigo's chromatic schemata as its object and which, in turn, gives rise to Vertigo as a film world. If the subject matter of film happens to coincide with the nature of the mental, then colour is not a reflection on the construction of the mental, but a reflection of its construction.

To sum up, I would say that colour works as a "force of coherence" compelling the viewer to accept the film as a world or as several interlocking film-worlds. I have borrowed the phrase "force of coherence" from George Steiner and I cannot but steal the complete sentence, unashamedly plagiarizing it over for my purposes. Steiner's subject matter is somewhat different: he is writing about Kierkegaard and considering the importance of his means of expression, his style, in relation to his philosophy. If we substitute "philosophy" with "film", insert "colour" where "language" stood and change "listener or reader" for "viewer" this is what we end up with:

A great film is always "stylish": that is to say that its impact on the viewer, the force of coherence which it generates, its music of persuasion, are necessarily cognate with its performative means (those of colour). ${ }^{(6)}$

I suggest that Vertigo has four different chromatic schemata and that they are deftly woven together to form the complete fabric of the film. These schemata are at times coiled around each other like a cord or alternately, cast in isolation. The first chromatic schema is marked by the different hues and varying saturations that constitute the whole gamut of colours situated between deep mahogany reddish-browns and its complementary emerald greens (or better still, the same in German: giftgrün, meaning "poisonous green"). This schema supplies a background wash for the film's first part and later resurfaces encased in significant details or else appearing highly saturated or grossly overstated. If, as is the case here, a chromatic schema is composed of two colours, one or the other might be present, or they might appear together.

Gavin Elster's laden office, and later in his club, display this rich darkened mahogany red-brown that permeates everything around it: the panelling, the heavy furniture, the carpets, even the books strewn on Elster's office floor (pic. 1). ${ }^{(7)}$ In these scenes there is little evidence of its complimentary green. At Ernie's, on the other hand, the same colour schema works differently. The walls are imbued in much a more saturated magenta-ish red, highlighting the green trim of Madeleine's stole (pic. 2). Though these colours set the tone of the whole first part of the film by creating a domineering wash (sensed, for instance, in narrow, dark and barely passable alleyways or haunted hotels. They are also marked in details, be it, for instance, Madeleine's green trim mentioned earlier, her car, or Scottie's jumper. 
A chromatic schema can be hinted at or can be spelled out clearly. When Scottie admits to Judy that she reminds him of Madeleine we see her in a silhouette cast against the highly saturated and glowing giftgrün curtain followed by a "portrait" of her drowned in the same deathly colour (pic. 3). Its next occurrence is in the film's magnificent climax. Judy remerges out of the bath as Madeleine shrouded in a ghostly green cloud (pic. 4). Walking towards Scottie, the cloud evaporates and they are engulfed in the celebrated revolving kiss, "the most magical camera movement in the history of cinema", the "love scene per se" (the words are Chris Marker's).

This chromatic schema identifies and relates to one possible world or one set of narrative relations. It defies a simple label, but for sake of convenience one could call it the Thanatos, since it is associated with Elster's master plan involving the murder of his wife and Scottie losing his beloved Madeleine to the dead. It is one of the leitmotifs of the film and constitutes its most noticeable agenda: ghosts, death, return of the dead, return to the dead, mortality, anxiety, melancholia, suicide and murder. One can read as much or as little as one wants into the specific meaning or meanings of this schema yet it is all but impossible to deny that it betrays the main narrative drive of the film. Pervading Vertigo, it permeates the film like mists hovering an abandoned castle in a Gothic novel. Constantly on the move though never losing its grip, it opens up and closes in, momentarily evaporating only to re-appear, unabated. The pervasive nature of this schema is derived in part from the fact that it is not composed of definitive hues but a complete range commencing at the cyan-green and terminating at the complementary magenta-red. This overall effect is demonstrated in plate 2 .

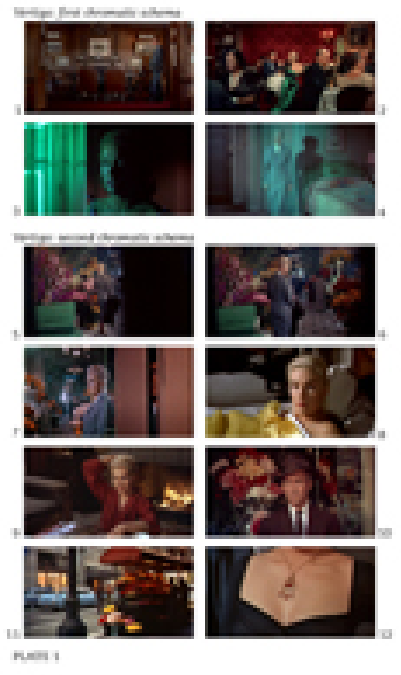

Figure 1: Plate 1

Although my primary concern here is the role colour plays in creating a film world, one cannot fail to mention the exemplary use of music in Vertigo and how it too is woven into the film, contributing to its sense of whole. The musical counterpart of this chromatic schema is conveyed by the use of extended "wandering" repetitions of brief phrases or else syncopated chords in the lower register. The music corresponding to this colour schema is atmospheric but not lacking sophistication. It creates a musical framework that can sustain flowering phrases and distinct themes, much like a wash that supports and defines all other colours.

The next chromatic schema is markedly different. Unlike the pervading wash that characterized the first schema this one tends to appear as chromatic outbursts. Its distinguishing form is red and yellow gushing out of the frame, typically as objects and most commonly in flowers. Right from the beginning these outbursts draw attention to themselves and consequently to their detached nature. They appear like shooting stars in the night sky: independent, defiant, vibrant, but short-lived.

Bursting out, breaking through and cutting off, these are the presiding tendencies of this chromatic schema. 
Severance and cleavage are constantly accentuated. Separate worlds and worlds apart, distinct from each other as red is from yellow: life and death, sleep and wakefulness, fire and water. Consider for example the first compelling evidence for this chromatic schema. Having followed Madeleine into a dreary alleyway, Scottie parks his car and enters an even darker passageway. Momentarily, the frame is completely black and then he slowly opens the door to the outpouring of colour, dominated by reds and yellows (pics. 5-7). Significantly, there is also a green reminiscent of the first chromatic schema.

The bursting out, breaking through and cutting off, characteristic of this chromatic schema, are also evidenced in its other manifestations. Scottie has followed Madeleine to a grey cemetery where she is visiting Carlotta's grave. Twice we see her through red and yellow flower patches that burst, momentarily, out of the scene. Scottie's first sighting of Judy is also marked by red and yellow flowers (pic. 10) and so too her "Madeleinization" at Ransohoffs' (pic. 11). Less sporadic and much more articulate is the appearance of this chromatic schema at Scottie's flat after he has saved Madeleine from drowning in San Francisco bay. After a fade to black in which he presumably entertains her in his bed, Madeleine wakes up startled by the phone ringing. She is naked in his bed, covered by a yellow blanket (pic. 8). He offers her his red bathrobe with which she emerges, somewhat stupefied, from his bedroom. Her clothes are hung to dry in his kitchen. Scottie offers her some tea and then makes her sit by the warmth of the yellow fireside (pic. 9). The colours speak the divisions; they mark the world of sleep and the world of wakefulness and the harsh divide between them. They emphasize the warmth of the fire with the brutality of the cold and wet bay. Yet, as in the flower shop, the chromatic remnants of a different world, that of Thanatos, lingers on. The scene is cut short (it is in the nature of this chromatic schema to be cut short) by yet another phone call from Gavin Elster.

If the first chromatic schema was aligned with Thanatos, this one is libidinous. Its vibrancy heralds love, falling in love, warmth, affection and life affirmation; but the sporadic and explosive nature of its appearances designates libido as fleeting and untenable. If it is love (which I think it is) it is far removed from the romantic ideal of love in the Sturm und Drang manner, that is as a storm of passions overpowering a highly emotional individual. This is not entirely absent from this schema, except that it is inserted contrapuntally by means of the music, which in the vicinity of this schema constantly veers towards exaggerated romanticism (Bernard Herrmann never concealed the influence of Mahler and Bruckner on his scores).

There is something uncanny in this relationship right from the start and not surprisingly, a number of commentators have detected quite a few elements of perversion in the story of an obsessive man and his voyeuristic attraction towards a masochistic woman. It is not my intention to comment on this, merely to identify the diegetic dimension of this chromatic schema and to draw some conclusions on its nature given its portrayal. Standing apart or bursting forth is as crucial here as its untimely disappearance. Unlike the spectral use of the first chromatic schema, this one makes use of distinct, easily recognized colours, mostly reds and yellows. It comes over not as a wash or a floating mist. Instead its presence is highly articulated and well-defined, almost like a flag or a standard-bearer signalling the presence of a new mood. This schema's treatment of tension has a distinct musical nature, aligning itself readily with the film's actual score. Accordingly its characteristic musical expression is not atmospheric but descriptive, as in the phrases and motifs that point to well-defined elements. A case in point is the use of Madeleine's theme soaring above the "atmospheric" syncopated chords mentioned previously in relation to the first chromatic schema.

The tension evident in this schema is erotic and the chromatics spell out the progression of desire: from desire dawning as if from nowhere, to its identification of an object and the ensuing sense of thrill tinted by an ominous feeling of risk and peril. Towards the end of the film, having brought Madeleine back from the dead, Vertigo's Orphic hero consummates his love, off screen, and perhaps, just perhaps, begins to love her as Judy. But the Gods intervene and make her wear Carlotta's fateful necklace, that souvenir of a killing implanted so seductively on her breast, so much so that only the mirror, the other gaze, can show its real nature. The necklace she won as a trophy from Elster for playing the temptress is now tempting fate to play with her life. The necklace, mind you, is in ruby and gold - red and yellow again (pic. 12).

Vertigo's third chromatic schema relies less on hues and more on the intensity of contrasts. We witness this in the juxtaposition and conjunction of contrasting and clashing hues - mostly black, white and red. Sometimes these contrasts appear together in one shot or in one scene. Alternatively, the same chromatic effect may be brought about not simultaneously but rather as a succession of images in time. The emblematic appearance 
of this schema is the scene on Scottie's front porch, after he has tracked her back to his own place from her apartment. The confident deployment of contrasting hues within well-defined patches of black, white and red induce a sense of stark clarity evident in the scene as a whole. Madeleine gravitates round a stark red door, dressed in a brilliant white coat punctuated by black gloves, black scarf and a large black handbag (see first row of plate 3). A similar set of chromatic relationships comprising this schema is apparent in what is surely one of the most beautiful tableaux in the film: Madeleine at the foot of the Golden Gate Bridge contemplating her impending jump into the San Francisco Bay (see sixth row of plate 3). Solid areas of colour (such as red and black) defined by highly articulated contours sit next to each other or hover about (such as the large white clouds).

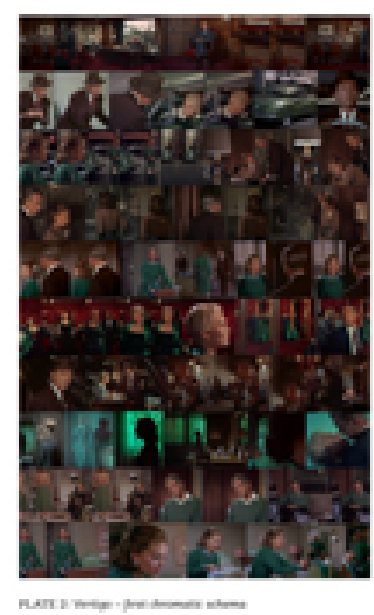

Figure 2: Plate 2

The most explicit use of contrast representative of this schema is evident when it is exercised as a transition between light and dark, between white and black. This duality dominates the visit to the Agrosy Bookshop where Pop Liebel tells Scottie and Midge about "Mad Carlotta". As he recounts the story the shop gradually darkens, but the moment they are outside we see the light inside being turned on and the black is instantly superseded by white. Thus a chromatic schema can manifest itself both through space and through time (see third row of plate 3). It would be possible to enumerate many other instances of this chromatic schema and in all of them clarity and determination dominate.

The creative use of grey constitutes an important chromatic element that dwells within this schema, albeit more at its periphery. Madeleine's famous grey dress, the one she wears just before she dies and in which she is later "re-born" is the foremost example. A similar grey dress evokes the departed Madeleine twice at Ernie's (where Scottie first saw her) and at Ransohoffs, a gallery of grey dresses is paraded before Scottie and Judy until the right one crops up. This also the grey of tombstones, church walls and museum portals (see last row of plate 3 ).

The recurring motif associated with this chromatic schema is that of determination, in both senses of the word: as firmness of purpose and resolute intention to carry out an action. Both senses are upward movements, one of the will the other of the faculty of knowledge. Clarity, lucidity and contrast enable action, whether physical or mental and both imply the world of action. Discernable contrasting colours enable clarity of purpose which is in turn a prerequisite for accomplishing a goal. The same clarity as an epistemological idea is the most fundamental account of knowledge: the ability to distinguish, to discern, to determine. The Tree of Knowledge, eating of whose fruit irrevocably changed the destiny of mankind, is, we shall recall, the tree of knowing, of discerning right from wrong. Scottie as an investigator seeks the truth, he looks for clues, for hints, for keys; he also looks for missing people. He tries to piece together parts of a puzzle that will 
make a complete picture. This drive is a basic world in Vertigo and not only Scottie partakes in it. Like the two meanings of the word "determination", the other side of this drive is set in volitional terms. The music associated with this schema is highly evocative of its underlying nature. Great surges enhanced by "real" crashing waves are one example, as are the jarring chords that accompany Madeleine's plunge into the ocean. Plate 3 summarizes some of the instances of this chromatic schema.

These three chromatic schemata are the structural unifying elements of the film. They are the basic elements that enable us to experience Vertigo as a coherent film-world irrespective of the plot. They are both antecedent to the narrative and sequential to it, thus going far beyond it.

The fourth chromatic schema is not, strictly speaking, a schema but its apparent opposite. It is the condition of the complete breakdown of colour and accordingly the absence of any coherence. A world can make sense if it has a unifying element that structures and connects all the disparate elements that comprise its parts. A world collapsed is a world whose unifying element ceases to function. This is what happens to Scottie at the beginning of the second part of the film, what Chris Marker has called "the other side of the mirror". (8) His nervous breakdown, conveniently diagnosed for us as acute melancholia coupled with guilt, is instigated by a richly symbolistic nightmare. Bouquets come apart, museum portraits come alive, open graves swallow up helpless victims causing them to fall into an endless void. Definitely enough material to keep a host of psychiatrists busy for quite some time; and yet, the most telling feature of this nightmare, at least philosophically, are not its symbolic or psychological details, but the fact the whole nightmare sequence is nothing but the breakdown of colour coherence in a vertiginous descending motion.

Colour, so far, has been rich in detail and highly subtle. Minute gradations combined to make a penetrating and sustaining wash on which chromatic flourishes conveyed fragile complexities rich in nuance and detail. None of this survives into the nightmare. The nightmare sequence is comprised of intermittent flashes of single tones, seemingly without sensible connection between them. The animated sequence shows Carlotta's bouquet coming apart, but most importantly, losing its colour in the process. The erratic and chaotic flashes of colour continue throughout until their complete obliteration into a black figure falling rapidly onto a white vacuum. Just before the moment everything turns into blank white, the moment before the film annihilates itself, Scottie wakes up in the throes of terror and crushed by anguish. The next moment he is in a mental hospital having Mozart administered to him in the form of music therapy. One suspects that had that little black figure falling into the white void disappeared completely, the next scene would have been either Scottie's real funeral grave, or - what amounts to the same - the film's end titles. (See plate 4).

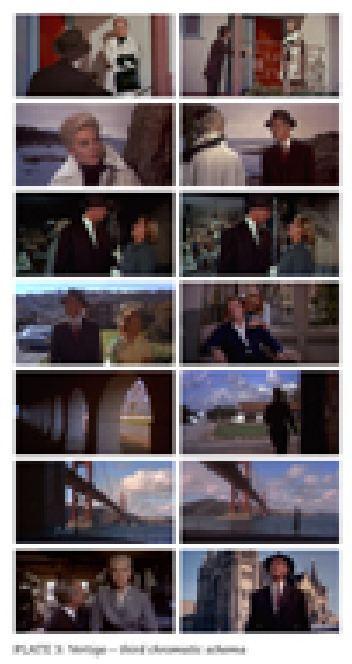

Figure 3: Plate 3

At times one feels that the nightmare sequence is the only scene in this masterpiece that has somewhat 
dated. The animation sequence, for instance, certainly isn't endowed with the rest of the film's timelessness. And yet, given the film's overall chromatic effect the portrayal of breakdown as a disintegration of colour is inspired, especially when it is coupled with its highly expressive music of orchestral surges culminating in a blearing dissonant climax. This supposed fourth schema works in two ways. As an antithesis it serves to distinguish and outline the boundaries and the richness of the previous three. The periodic flickering of saturated monochromatic hues is annoying and not only signals danger (like the lights of a police car siren); but also destruction. Destruction of the main character's well-being, his personality and his mental equanimity. And, moreover, a destruction of the film's idiomatic structure. The camera ceases to observe events, whether from an objective or subjective point of view. It positions itself outside of time and space so as to reside beyond the mimetic and inside the mental. This involves a paradox since no camera can actually be positioned there, certainly not in the sense that it can position itself observing space and time. Film has never been deterred from trying to portray what one could call the internal and there are many different approaches on how to create the transition from a mimetically possible depiction of space and time to the mimetically impossible depiction of an internal world. In Vertigo, this transition is clearly marked by the film's idiomatic use of colour, hence the colour breakdown in the nightmare sequence.

Worlds are created and worlds come apart. Worlds are deemed complete or embryonic. Worlds evolve, transform and change. However we choose to talk of worlds, there seems to be no possibility of ignoring the need to recognize their marks of coherence, their internal signifiers that serve to agglutinate their disparate elements. Without understanding how these elements are put together we would be unable to say that one world evolves and carries itself through different stages of its metamorphosis. What sense would there be in saying that a world had come apart, if we knew not how to identify it; or, how would we recognize the boundaries of one world if it did not avail itself as an organized structure with a sensible mark of coherence.

The role colour plays in the creation of a film world creates a unique dialectics. In one sense colour sets the necessary conditions for the evolution of a film world, in another sense, it provides markers for recognizing these self-determined entities. A ray of light bursting out of a film projector heralds the creation of a world, but in itself, this is nothing. It is only when the play of colour drawn by strips of film passing rapidly in front of that ray of light, that things can begin to take form. This in itself is not a world yet, these disjointed events need to be combined for a veritable world to take form. This is the dialectical quality of colour in film. As light, it anticipates the creation of a film world, as a syntactic element it indexes this world, enabling the completion of its creation.

This dialectical nature of colour does not only reside in its ontology. We noted previously the distinction between the perception and the apperception of colour. The former refers to the ability of light to cause sensory stimulation, while the latter placed itself as a cognitive act located in between this sensory experience and intellectual cognition. For a film to move us, it must make sense and no doubt this is achieved only if we tap into its richly coded language. Every object, every gesture, every word, every feature of film is endowed with some sort of meaning and likewise its use of colour forms one node in a dense semantic field. An interpretation of film relies on our ability to move about freely in this field. The basic contention of this essay is that in order to fully appreciate a film it is necessary to recognize its more rudimentary, pre-semantic structures which I have suggested are syntactic in nature and constitute the formation of a film world. Colour was offered as one such apperceptive building block, located above the mere sensory perception but beneath, so to speak, intellectual-semantic cognition. The apperception of film's colour schema reduplicates the act of perception at a higher level. Whereas the perceiving eye has visual stimulus as its object, for the apperceiving mind considers the chromatic schema as its object.

The dialectical nature of colour and light as creative elements in cinema has been magnificently caught in film, in the opening, pre-title sequence, of Ingmar Bergman's Persona. This curious visual mediation on life and death, on creation and sacrifice, lasts for five and a half minutes. The film begins with a darkened screen, in which a small patch of light starts to increasingly glow, until it blazes on the screen. Just before an arc light ignites, we realize that we are inside the lamp chamber of a film projector. Next, following this moment of the creation, we hear the sound of a filmstrip passing through a projector and see an extreme close up of the spooled film strip running briskly along its tracks. Alternate frames of white and black fill the whole screen. With light, cinema comes into being, first in the form of a spool leader and then in a short animation sequence. The rest of this meditation presents striking images of sacrifice and death. Film as a 
medium makes a few other notable appearances in Persona, the most important of which to our discussion is the complementary closure of the film. There we see a filmstrip inside a projector running out of its track, followed by the darkening of the screen as the two rods that generate the arc light part from each other. Judging from the published script of Persona (which does not follow the film frame by frame and is in many respects quite independent of it), Bergman's meditation concerns, among other things, the power of film light to generate a seemingly coherent world. "I imagine the transparent ribbon of film rushing through the projector" he begins the script. "Washed clean of signs and pictures, it produces flickering reflected light from the screen". As the light begins to "establish itself and thicken" and amidst "incoherent" sounds and words, an image takes form, the image of a face, "its eyes locked into those of the spectator. The face of Sister Alma". ${ }^{(9)}$ The face, or perhaps the mask, the persona, is here the outward sign of coherence underneath which mystery lies. The face is to the personality, what colour is to film.

I have argued that colour (broadly defined) is a crucial element in the formation of a film's integrity, a film's power to create a sense of whole from its disparate constituents. The ability to meaningfully combine these elements so as to create a congruent and coherent world resides at the film's syntactic level and I have sought to demonstrate this process through a detailed analysis of Vertigo's chromatic structure. The purpose of this approach is not only to uncover a method by which it will be possible to expose the underlying frameworks of films but also to lay the groundwork for a meaningful philosophical exposition of such films. Vertigo is a case in point: a narrative analysis begets one possible, but limiting, vision of the film. Accessing the deeper structures of Vertigo, as I believe a sensitivity to its exemplary use of the cinematic medium will reveal, enables us to examine the film's unique contribution in understanding pivotal philosophical issues. The next question this suggested approach needs to address is, given this understanding of film, what specific world does it give rise to and what unique insights may be gleaned from it - but that is an issue all to itself.

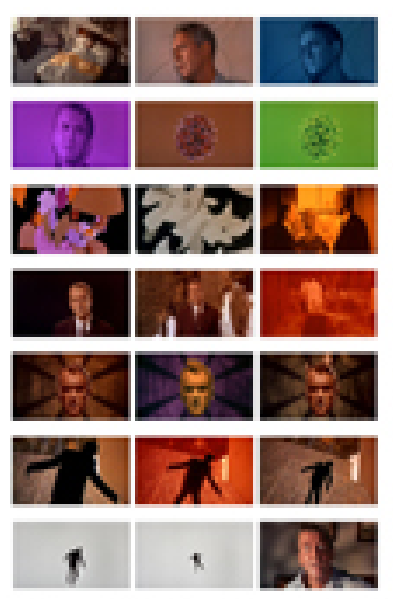

Figure 4: Plate 4

\section{Notes}

1. Jean Renoir, "Marcel Pagnol" in Gilbert Adair (ed.), Movies (Harmondsworth: Penguin Books, 1999), p. 340

2. Paul Klee, The Diaries of Paul Klee, (entry for April 16, 1914), quoted in Robert Kudielka, Paul Klee: 
The Nature of Creation (London: Hayward Gallery 2002), p. 30

3. Quoted by Sam Rohdie in Antonioni (London: BFI Publishing, 1992), p. 159

4. John Gage, Colour and Meaning: Art, Science and Symbolism (London: Thames \& Hudson, 1999), p. 56

5. Ibid., p. 30

6. See "On Kierkegaard" in No Passion Spent (London: Faber and Faber, 1996), p. 256

7. All pictures are from Alfred Hitchcock's Vertigo (1958), () Universal Studio Inc.

8. "A Free Replay (Notes on Vertigo)" in John Boorman and Walter Donohue, Projections 4 1/2 (Faber \& Faber: London, 1995)

9. Ingmar Bergman, Persona and Shame (London: Marion Boyars, 1966), p. 23

\section{Author Information}

Ornam ROTEM is lecturer in comparative philosophy at Tel Aviv University's School of Philosophy. His interests include philosophical expositions of film and is currently in the process of writing a book on film and philosophy summing up his research in the field. He has designed and taught courses dealing specifically with philosophical readings of film and courses on Buddhism and film. 